Detschew, Vesselin; Suchi, A.; Bopple, M.:

\title{
EEG - Analyse für das HP Component Monitoring System
}

\author{
Zuerst erschienen in: Biomedizinische Technik = Biomedical Engineering. - Berlin [u.a.] : \\ de Gruyter. - 43 (1998), s1, S. 94-95. \\ Erstveröffentlichung: 1998 \\ Datum Digitalisierung: $\quad 23.10 .2009$ \\ ISSN (online): $\quad$ 1862-278X \\ ISSN (print): $\quad$ 0013-5585 \\ DOI: $\quad$ 10.1515/bmte.1998.43.s1.94 \\ [Zuletzt gesehen: 31.07.2019]
}

„Im Rahmen der hochschulweiten Open-Access-Strategie für die Zweitveröffentlichung identifiziert durch die Universitätsbibliothek IImenau."

"Within the academic Open Access Strategy identified for deposition by IImenau University Library."

„Dieser Beitrag ist mit Zustimmung des Rechteinhabers aufgrund einer (DFGgeförderten) Allianz- bzw. Nationallizenz frei zugänglich."

"This publication is with permission of the rights owner freely accessible due to an Alliance licence and a national licence (funded by the DFG, German Research Foundation) respectively."

\section{DFG}

Nationallizenzen 


\title{
EEG - Analyse für das HP Component Monitoring System
}

\author{
Detschew, V.*, Suchi, A.**, Bopple, M.** \\ * Technische Universität Ilmenau, Institut BMTI, Postfach 100565, 98684 Ilmenau \\ ** Hewlett Packard Medzinelektronik, POB 1427, 71004 Böblingen
}

Ein im Patientenmonitoring bisher sehr wenig genutztes biologisches Signal ist das EEG. Das liegt daran, daß die Ableitbedingungen im allgemeinen bedeutend schwieriger sind als bei anderen Vitalgrößen. Andererseits ist sehr viel Erfahrung und Wissen notwendig, um aus dem EEG diagnostisch relevante Informationen $\mathrm{zu}$ erhalten. Aus diesen und verschiedenen weiteren Gründen gibt es am Markt auch nur sehr wenige Patientenmonitoringsysteme, die die Ableitung von EEG unterstützen.

Im Beitrag wird die Entwicklung einer „erweiterten Funktionalität" außerhalb des Patientenmonitors beschrieben. Ausgehend von den gerätetechnischen Gegebenheiten, d.h. am Markt verfügbaren EEG - Monitoringsystemen, und den Möglichkeiten der rechnergestützten Analyse von Biosignalen, wurde ein Konzept entwickelt und umgesetzt. Die Implementierung der Analyseverfahren orientierte sich stark an den Wünschen von Anästhesiologen und Intensivmedizinem, um von Anfang an eine möglichst hohe Akzeptanz zu errei- chen. Die Software läuft auf einem eigenständigen Rechner (PC) und bezieht ihre Daten vom Hewlett Packard Component Monitoring System (CMS).

Die wesentlichen Eigenschaften des Systems sind:

- Allgemeine Funktionalität: Hier sind alle Eigenschaften der Software gemeint, die für den Umgang mit dem System notwendig sind und vom Anwender vorausgesetzt werden. Dazu zählen die Arbeit mit Dateien, Druckern und Hilfefunktionen. Allgemeinere Spezifikationen, wie Anordnung der Menüs o.ä., werden hier nicht extra aufgeführt, da das Prinzip des "look and feel" einer Windows Anwendung verwirklicht werden sollte.

- Konventionelle Analyse und Darstellung: Es wurden die Methoden der Spektralanalyse sowie die daraus abgeleiteten Parameter: spektrale Eckftequenz SEF90/95, spektrale Medianfrequenz SMF, mittlere dominante Frequenz MDF, Spitzen-LeistungsFrequenz PPF, absolute und relative Bandleistungen

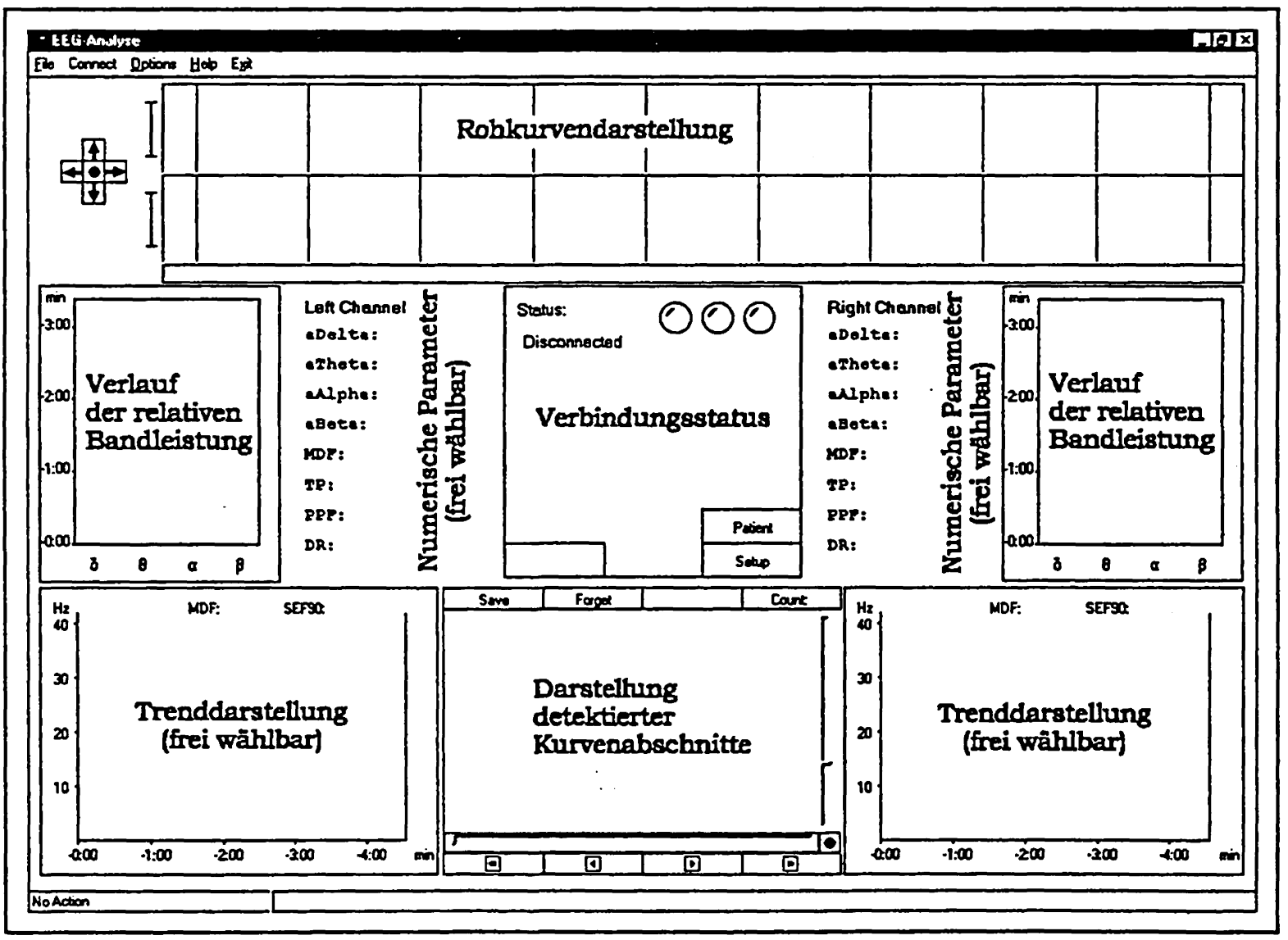


in den für die EEG-Auswertung üblichen Frequenzbändern usw. implementiert. Weiterhin wurden Darstellungen der Kurven und der numerischen Parameter verwirklicht, wobei eine nutzerabhängige Konfiguration angestrebt wurde.

- Erweiterte Funktionalität: in der ersten Fassung der Software sind Ansätze zur Musterdetektion implementiert und anhand von Datenmaterial validiert. Es wurde von der Detektion von epileptischen Spikes bzw. Spike-Wave-Komplexen ausgegangen. Die Software soll jedoch nur den Schritt der on-line Detektion von "verdächtigen" Abschnitten erfüllen und diese einer späteren visuellen off-line Inspektion zuführen.

Die Applikation wurde auf einem Windows NT Rechner in Borland Delphi unter Nutzung von Standard Klassen sowie unter Verwendung eigener Signalanalyse - Komponenten realisiert.

Das HP CMS ist zur Übertragung von Daten mit einer Standard - RS232 Schnittstelle ausgestattet. Diese kann mit maximal 38400 Baud betrieben werden. Die Übertragungsrate führt zu einer Begrenzung der Bandbreite der zu übertragenden Werte. Da sich die vorliegende Anwendung nur auf das Roh - EEG Signal und die EEG-Parameter beschränkt, stellt das für diese Arbeit kein Problem dar. HP definiert für den Patientenmonitor das sogenannte MECIF (Merlin Computer Interface) Protokoll. Dieses Protokoll ermöglicht den Datenaustausch zwischen Server (CMS) und Client (Computer) durch den Austausch von Botschaften. Werden Daten übermittelt, liegen diese in einem speziellen Format, dem SPI (Standard-Parameter-Interface) - Format vor. Es wurde ein Treiber zur Verfügung gestellt, der sämtliche Aufgaben der Kommunikation abdeckt und in entsprechender Form der Analyse - Software zugänglich macht.

Beim Design der Oberfläche (Abb.) wurde darauf geachtet, $\mathrm{daß}$ nicht $\mathrm{zu}$ viele und überflüssige Bedienungselemente vorhanden sind. Die Hauptfunktionalität ist ohne besondere Kenntnisse sofort zu erreichen. Von Windows bekannte Bedienungselemente (Menü, Buttons, Statusleiste) ermöglichen den einfachen Zugriff auf die Funktionen und geben den Systemstatus wieder. Die Oberfläche wurde so gestaltet, daß sie intuitiv bedient und möglichst einfach abgelesen werden kann. Die Rohkurven werden im oberen Bereich dargestellt. Die Darstellung ist sowohl in der Amplitude, als auch im Zeitmaßstab beliebig skalierbar, so daß den Anforderungen jedes Benutzers entsprochen werden kann. Als weiterer fest vorgegebener Parameter wird der Verlauf der relativen Bandleistung dargestellt. Alle Kanalbezogenen Parameter sind auf der jeweiligen Bildschirmhälfte angeordnet. um so eine einfache Zuordnung der berechneten und angezeigten Parameter zu der Ableitregion herstellen zu konnen. Die implementierten Parameter sind in zwei Formen darstellbar.

- Numerische Darstellung: Aus allen berechneten Parametern lassen sich maximal acht zur numeri- schen Darstellung auswählen. Diese werden dann (je nach Länge der FFT) im Sekunden- bzw. Zweisekunden- Takt aktualisiert.

- Zusätzlich lassen sich zwei Parameter als Trend darstellen. Dabei erfolgt die grafische Darstellung des Verlaufes des entsprechenden Parameters über die letzten 4 Minuten.

In der Implementierung der spektralen Analyse wurde auf vorhandene Softwareobjekte (Komponenten) zurückgegriffen. Das Leistungsspektrum basiert auf einer Implementierung der FFT. Um eine konsistente Spektraldichteschätzung zu erhalten, wurde ein HanningFenster nach der Spektrenberechnung angewendet. Weiterhin werden als Ausgabeparameter neben dem kumulativen Leistungsspektrum die Peak-PowerFrequenz und die mittlere dominante Frequenz berechnet. Aus dem kumulativen Spektrum lassen sich sofort die Gesamtleistung und die absoluten Bandleistungen extrahieren. Ebenso einfach ist dann die Berechnung der relativen Bandleistungen. Als weitere Parameter werden dann die Delta Ratio, spektrale Medianfrequenz und die spektrale Eckfrequenz aus dem Leistungsspektrum berechnet. Der für die Kurvenglättung eingesetzte adaptive Mittelwert arbeitet mit einem KonstantenController wobei die Konstante wählbar ist.

Der Ansatz zur Detektion von Instationaritäten wurde mittels adaptiver Momentanleistung realisiert. Dieser Ansatz kann unter bestimmten Bedingungen die Detektion von Spikes und Spike-Wave-Komplexen sicher leisten. Auf den Verlauf der Momentanleistung wird dann ein Schwellwert angewendet, dessen Höhe empirisch ermittelt wurde. Es wurden alle Ereignisse im Test - EEG detektiert, die mit einer hochfrequenten Signalleistung behaftet waren. Ein weiterer Ansatz nutzt das Verfahren der adaptiven Quantilwertschätzung. Dieses Verfahren läßt sich jedoch nicht sinnvoll als eigenständige Detektion anwenden, da nur Bereiche detektiert werden, deren Amplitude außerhalb eines vorgegebenen Bereiches liegt. Es läßt sich allerdings als Erweiterung des erstgenannten Verfahrens ansehen, indem der im ersten Ansatz als konstant angenommene Grenzwer adaptiv berechnet wird.

\section{LITERATUR:}

[1] Suchi, A.: Entwicklung eines EEG-Analysesystems für das HP Component Monitoring System. Diplomarbeit, TU Ilmenau 1997

[2] Hewlett Packard: HP CMS 'Merlin', interne Spezifikation

[3] Grießbach, G. et al.: Dynamic description of stochastic signal by adaptive momentary power and momentary frequency and ist application in analysis of biological signals. Medical \& Biological Engineering \& Computing 32 (1994) 